\title{
ISHIKAWA AND MANN ITERATION METHODS FOR NONLINEAR STRONGLY ACCRETIVE MAPPINGS
}

\author{
M.O. OSILIKE
}

Let $X$ be a real Banach space with a uniformly convex dual, $X^{*}$, and let $C$ be a nonempty closed convex and bounded subset of $X$. Let $T: C \rightarrow C$ be a strongly accretive and a continuous mapping. For any $f \in C$, let $S: C \rightarrow C$ be defined by $S x=f+x-T x$ for each $x \in C$. Then, the iteration process $x_{0} \in C$,

$$
x_{n+1}=\left(1-\alpha_{n}\right) x_{n}+\alpha_{n} S x_{n}, n \geqslant 0
$$

under suitable conditions on the real sequence $\left\{\alpha_{n}\right\}_{n=0}^{\infty}$ converges strongly to a solution of the equation $T x=f$ in $C$. Furthermore, if $T$ is strongly accretive and Lipschitz with Lipschitz constant $L \geqslant 1$ then the iteration process $x_{0} \in C$,

$$
x_{n+1}=\left(1-\alpha_{n}\right) x_{n}+\alpha_{n} S\left[\left(1-\beta_{n}\right) x_{n}+\beta_{n} S x_{n}\right], n \geqslant 0,
$$

under suitable conditions on the real sequences $\left\{\alpha_{n}\right\}_{n=0}^{\infty}$ and $\left\{\beta_{n}\right\}_{n=0}^{\infty}$ converges strongly to a solution of the equation $T x=f$ in $C$. Explicit error estimates are obtained.

\section{INTRODUCTION}

Let $X$ be a real Banach space. A mapping $T$ with domain $D(T)$ and range $R(T)$ in $X$ is said to be accretive (see for example $[2,11,16,23]$ ) if the inequality,

$$
\|x-y\| \leqslant\|x-y+t(T x-T y)\|
$$

holds for each $x, y$ in $D(T)$ and for all $t>0 . T$ is said to be $m$-accretive if $T$ is accretive and $(I+r T)(X)=X$ for all $r>0$, where $I$ denotes the identity operator on $X$. If $X=H$, a Hilbert space, the accretive condition (1) is equivalent to the monotonicity of $T$ in the sense of Browder [3] and Minty [20]. The accretive operators were introduced independently in 1967 by Browder [2] and Kato [16] and the firm connection of this class of operators with the existence theory for nonlinear equations of evolution in Banach spaces is now well-known (see for example, $[6,13,16,19]$ ). An

Received 18 November 1991

Copyright Clearance Centre, Inc. Serial-fee code: $0004-9729 / 92 \$ A 2.00+0.00$. 
early fundamental result in the theory of accretive operators due to Browder [5], states that the initial value problem,

$$
\frac{d u}{d t}+T u=0, \quad U(0)=U_{0}
$$

is solvable when $T$ is locally Lipschitzian and accretive on $X$. Utilising the existence result for (2), Browder [5] proved that if $T$ is locally Lipschitz and accretive on $X$, then $T$ is $m$-accretive, so that for any $f$ in $X$, the equation,

$$
x+T x=f
$$

has a solution in $X$. Recently, Ray [23] gave an elementary proof of this result by employing a fixed point theorem of Caristi [7]. In [19] Martin proved that (2) is solvable if $T$ is continuous and accretive, and utilising the result he proved that if $T$ is continuous and accretive, then $T$ is $m$-accretive. Zarantonello [27] also proved that if $H$ is a Hilbert space and $T$ is an accretive and Lipschitzian mapping of $H$ into itself, then the equation (3) has a unique solution in $H$.

For a Banach space $X$, let $J$ denote the normalised duality mapping from $X$ to $2^{X^{*}}$ given by

$$
J x=\left\{f^{*} \in X^{*}:\left\|f^{*}\right\|^{2}=\|x\|^{2}=\left\langle x, f^{*}\right\rangle\right\}
$$

where $X^{*}$ denotes the dual space of $X$ and $\langle$,$\rangle denotes the generalised duality pairing.$ It is well-known that if $X^{*}$ is strictly convex, then $J$ is single-valued and if $X^{*}$ is uniformly convex, then $J$ is uniformly continuous on bounded sets (see for example, $[6,26])$.

In terms of the duality mapping, a mapping $T$ with domain $D(T)$, and range $R(T)$ in $X$ is accretive (see for example, $[2,16,23]$ ) if for each $x, y$ in $D(T)$, there exists $j \in J(x-y)$ such that,

$$
\langle T x-T y, j\rangle \geqslant 0
$$

A strong notion of accretiveness has been defined. A mapping $T$ with domain $D(T)$ and range $R(T)$ in $X$ is said to be strongly accretive with constant $k$ (see for example, $[1,21,12])$ if for each $x, y$ in $D(T)$, there exists $j \in J(x-y)$ such that

$$
\langle T x-T y, j\rangle \geqslant k\|x-y\|^{2}
$$

Without loss of generality we may assume $k \in(0,1)$. Strongly accretive mappings have been studied extensively by various authors (see for example, $[1,2,6,12,21,22]$ ). In [2] Browder proved the following result. 
Theorem B. Let $X$ be a Banach space and let $T: X \rightarrow X$ be a Lipschitz and strongly accretive mapping. Then $T$ maps $X$ onto $X$.

An obvious consequence of Theorem B is that for each $f$ in $X$, the equation

$$
T x=f
$$

has a solution in $X$. Recently, Morales [21] generalised the result of Theorem B to continuous strongly accretive mappings.

Methods for iteratively approximating solutions of equations (3) and (5) (when they are known to exist) have been investigated by several authors (see for example, $[8,9,11,12,14])$. We consider the two fixed point iteration methods given by the following:

(A) The Ishikawa Iteration Process. (See for example $[15,25]$ ) defined as follows:

For $C$ a convex subset of a real Banach space, and $T$ a mapping of $C$ into itself, the sequence $\left\{x_{n}\right\}_{n=0}^{\infty}$ is defined by

$$
\begin{aligned}
x_{0} & \in C, \\
x_{n+1} & =\left(1-\alpha_{n}\right) x_{n}+\alpha_{n} T y_{n}, n \geqslant 0, \\
y_{n} & =\left(1-\beta_{n}\right) x_{n}+\beta_{n} T x_{n}, n \geqslant 0
\end{aligned}
$$

and $\left\{\alpha_{n}\right\}_{n=0}^{\infty},\left\{\beta_{n}\right\}_{n=0}^{\infty}$ satisfy $0 \leqslant \alpha_{n} \leqslant \beta_{n}<1$ for all $n, \lim _{n \rightarrow \infty} \beta_{n}=0$ and $\sum_{n=0}^{\infty} \alpha_{n} \beta_{n}=$ $\infty$; and

(B) The Mann Iteration Process. (See for example $[18,25]$ ) which is similar to the iteration process (A) but with $\beta_{n}=0$ for all $n \geqslant 0$, and different conditions placed on $\left\{\alpha_{n}\right\}_{n=0}^{\infty}$.

The iteration processes described in (A) and (B) have been used extensively by several authors for approximating solutions of several nonlinear operator equations in Banach spaces (see for example, $[9,10,11,12,14,15,18,22,25])$. In [25] Rhoades compared the performance of these two iteration processes, and showed that even though they are similar, they may exhibit different behaviours for different classes of nonlinear mappings. In particular, there are examples where the Mann iteration process may fail to converge and the Ishikawa process converges. Also, there are problems for which the Mann process converges and the Ishikawa process does not. Furthermore, for the case where the two processes converge, the rate of convergence may differ. (For a detailed comparison of the two methods, the reader may consult [25]). Thus, it is of interest to examine the behaviour of the two processes for any given class of nonlinear mappings.

We note here that the Ishikawa iteration process was actually introduced in [15] to approximate a fixed point of Lipschitz pseudo-contractive maps in Hilbert space. For 
$C$ a compact subset of a Hilbert space, and $T: C \rightarrow C$ a Lipschitz pseudo-contractive mapping, it is still an open question whether or not the Mann iteration process converges to a fixed point of $T$.

For the operator equation (3) Dotson [14] proved that if $T: H \rightarrow H$ is monotone and Lipschitz with Lipschitz constant 1, (in this case $T$ is called nonexpansive in the terminology of Kirk) an iteration process of the Mann-type converges strongly to the unique solution of the equation. This result has recently been extended in a series of papers by Chidume $[8,9,11]$ to the case where $x$ is now any real Banach space with a uniformly convex dual $X^{*}$, and $T$ is accretive and continuous.

For the operator equation (5), Chidume [12, Theorem 1] recently proved that if $X=L_{p}, p \geqslant 2$, and $T: X \rightarrow X$ a strongly accretive and Lipschitz mapping with Lipschitz constant $L \geqslant 1$, then an iteration process of the Mann-type converges strongly to a solution of the equation.

It is our purpose in this paper to examine the Ishikawa and Mann iteration processes for the operator equation (5) in Banach space much more general than $L_{p}$ spaces, $p \geqslant 2$. In particular our results will include all $L_{p}$ spaces for $1<p<\infty$. Let $X$ be a real Banach space with a uniformly convex dual $X^{*}$, and $C$ a nonempty closed, convex and bounded subset of $X$. Let $T: C \rightarrow C$ be continuous and strongly accretive. We prove that under suitable conditions on the real sequence $\left\{\alpha_{n}\right\}_{n=0}^{\infty}$, the Mann iteration process converges strongly to a solution of equation (5). Furthermore, if $T$ is strongly accretive and Lipschitz with Lipschitz constant $L \geqslant 1$, then the Ishikawa iteration process, under suitable conditions on the real sequences $\left\{\alpha_{n}\right\}_{n=0}^{\infty}$ and $\left\{\beta_{n}\right\}_{n=0}^{\infty}$, converges strongly to a solution of equation (5). For some particular choices of the real sequence $\left\{\alpha_{n}\right\}_{n=0}^{\infty}$, explicit error estimates are obtained.

\section{Preliminaries}

In the sequel we shall need the following remarks.

REMARK 1. In [24, p.89], Reich proved that if $X^{*}$ is uniformly convex, then there exists a continuous nondecreasing function,

$$
b:[0, \infty) \rightarrow[0, \infty)
$$

such that

$$
b(0)=0, \quad b(c t) \leqslant c b(t), \quad \text { for all } c \geqslant 1 \text {, }
$$

and

$$
\|x+y\|^{2} \leqslant\|x\|^{2}+2\langle y, j(x)\rangle+\max \{\|x\|, 1\}\|y\| b(\|y\|) .
$$

REMARK 2. Nevanlinna and Reich [22] have shown that for any given continuous nondecreasing function $b$ with $b(0)=0$, sequences $\left\{\lambda_{n}\right\}_{n=0}^{\infty}$ always exist such that

(i) $0<\lambda_{n}<1, n \geqslant 0$ 
(ii) $\sum_{n=0}^{\infty} \lambda_{n}=\infty$, and
(iii) $\sum_{n=0}^{\infty} \lambda_{n} b\left(\lambda_{n}\right)<\infty$.

If $X=L_{p}, 1<p<\infty$, we can choose any sequence $\left\{\lambda_{n}\right\}_{n=0}^{\infty}$ in $l^{\prime} \backslash l^{\prime}$ with $s=p$ if $1<p \leqslant 2$ and $s=2$ if $p \geqslant 2$.

For the rest of this paper, the Lipschitz constant of $T$ is denoted by $L(\geqslant 1)$ and the constant appearing in the definition of a strongly accretive mapping is denoted by $k \in(0,1)$.

\section{Main Results}

We prove the following theorems.

Theorem 1. Let $X$ be a real Banach space with a uniformly convex dual, $X^{*}$, and let $C$ be a closed convex and bounded subset of $X$. Let $T: C \rightarrow C$ be a continuous and strongly accretive mapping. For any $f$ in $C$, define $S: C \rightarrow C$ by $S x=f+x-T x$, $x \in C$. Let $\left\{\alpha_{n}\right\}_{n=0}^{\infty}$ be a real sequence satisfying

(i) $0<\alpha_{n}<1, n \geqslant 0$

(ii) $\sum_{n=0}^{\infty} \alpha_{n}=\infty$, and

(iii) $\sum_{n=0}^{\infty} \alpha_{n} b\left(\alpha_{n}\right)<\infty$.

Then, given any $f$ in $C$, the iteration process, $x_{0}$ in $C$,

$$
x_{n+1}=\left(1-\alpha_{n}\right) x_{n}+\alpha_{n} S x_{n}, \quad n \geqslant 0
$$

converges strongly to a solution of the equation, $T x=f$ in $C$.

Proof: The existence of a solution $x^{*}$ of $T x=f$ in $C$ follows from Morales [21] (see also [12]). Observe that $x^{*}$ is a fixed point of $S$ and since $T$ is strongly accretive, then for each $x, y$ in $C$,

$$
\begin{aligned}
\langle S x-S y, j(x-y)\rangle & =\|x-y\|^{2}-\langle T x-T y, j(x-y)\rangle \\
& \leqslant(1-k)\|x-y\|^{2} .
\end{aligned}
$$

Using inequality (6) and equation (7), we obtain

$$
\begin{aligned}
\| x_{n+1} & -x^{*}\left\|^{2}=\right\|\left(1-\alpha_{n}\right)\left(x_{n}-x^{*}\right)+\alpha_{n}\left(S x_{n}-x^{*}\right) \|^{2} \\
\leqslant & \left(1-\alpha_{n}\right)^{2}\left\|x_{n}-x^{*}\right\|^{2}+2 \alpha_{n}\left(1-\alpha_{n}\right)\left\langle S x_{n}-x^{*}, j\left(x_{n}-x^{*}\right)\right\rangle \\
& \quad+\max \left\{\left(1-\alpha_{n}\right)\left\|x_{n}-x^{*}\right\| 1\right\} \alpha_{n}\left\|S x_{n}-x^{*}\right\| b\left(\alpha_{n}\left\|S x_{n}-x^{*}\right\|\right) \\
\leqslant & \left(1-\alpha_{n}\right)^{2}\left\|x_{n}-x^{*}\right\|^{2}+2 \alpha_{n}\left(1-\alpha_{n}\right)\left\langle S x_{n}-x^{*}, j\left(x_{n}-x^{*}\right)\right\rangle \\
& \quad+\max \left\{\left(1-\alpha_{n}\right)\left\|x_{n}-x^{*}\right\|, 1\right\} \alpha_{n}\left\|S x_{n}-x^{*}\right\| \max \left\{\left\|S x_{n}-x^{*}\right\|, 1\right\} b\left(\alpha_{n}\right), \\
\leqslant & \left(1-\alpha_{n}\right)^{2}\left\|x_{n}-x^{*}\right\|^{2}+2 \alpha_{n}\left(1-\alpha_{n}\right)\left\langle S x_{n}-x^{*}, j\left(x_{n}-x^{*}\right)\right\rangle+M \alpha_{n} b\left(\alpha_{n}\right),
\end{aligned}
$$


for $M>0$, since $C$ is bounded.

Using inequality (8), we obtain

$$
\begin{aligned}
\left\|x_{n+1}-x^{*}\right\|^{2} & \leqslant\left(1-\alpha_{n}\right)^{2}\left\|x_{n}-x^{*}\right\|^{2}+2 \alpha_{n}\left(1-\alpha_{n}\right)(1-k)\left\|x_{n}-x^{*}\right\|^{2}+M \alpha_{n} b\left(\alpha_{n}\right) \\
& =\left[\left(1-\alpha_{n}\right)^{2}+2 \alpha_{n}\left(1-\alpha_{n}\right)(1-k)\right]\left\|x_{n}-x^{*}\right\|^{2}+M \alpha_{n} b\left(\alpha_{n}\right) \\
& \leqslant\left[\left(1-\alpha_{n}\right)^{2}+2 \alpha_{n}\left(1-\alpha_{n}\right)(1-k)+\alpha_{n}^{2}(1-k)^{2}\right]\left\|x_{n}-x^{*}\right\|^{2} \\
& +M \alpha_{n} b\left(\alpha_{n}\right) \\
& =\left[1-k \alpha_{n}\right]^{2}\left\|x_{n}-x^{*}\right\|^{2}+M \alpha_{n} b\left(\alpha_{n}\right) \\
& \leqslant\left[1-k \alpha_{n}\right]\left\|x_{n}-x^{*}\right\|^{2}+M \alpha_{n} b\left(\alpha_{n}\right) .
\end{aligned}
$$

Thus

$$
\left\|x_{n+1}-x^{*}\right\|^{2} \leqslant\left[1-k \alpha_{n}\right]\left\|x_{n}-x^{*}\right\|^{2}+M \alpha_{n} b\left(\alpha_{n}\right)
$$

Following the method of proof of Theorem 1 of [10], (see also [11]), we set $\rho_{n}=$ $\left\|x_{n}-x^{*}\right\|^{2}, \delta_{n}=k \alpha_{n}$, and $\sigma_{n}=M \alpha_{n} b\left(\alpha_{n}\right)$. Then inequality (9) reduces to

$$
\rho_{n+1} \leqslant\left[1-\delta_{n}\right] \rho_{n}+\sigma_{n}
$$

and as in [10] we obtain $\rho_{n} \rightarrow 0$ as $n \rightarrow \infty$, so that

$$
x_{n} \rightarrow x^{*} \text { as } n \rightarrow \infty \text {. }
$$

Corollary 1. Let $X=L_{p}, 1<p \leqslant 2$, and let $C, T$ and $S$ be as in Theorem 1. Let $\left\{\alpha_{n}\right\}_{n=0}^{\infty}$ be a real sequence satisfying

(i) $0<\alpha_{n}<1, n \geqslant 0$

(ii) $\sum_{n=0}^{\infty} \alpha_{n}=\infty$, and

(iii) $\sum_{n=0}^{\infty} \alpha_{n}^{p}<\infty$.

Then, for any $f$ in $C$, the iteration process, $x_{0} \in C$,

$$
x_{n+1}=\left(1-\alpha_{n}\right) x_{n}+\alpha_{n} S x_{n}, \quad n \geqslant 0
$$

converges strongly to a solution of the equation $T x=f$ in $C$.

Proof: The existence of a solution $x *$ of $T x=f$ in $C$ follows from Morales [21]. Remark (2), conditions (ii) and (iii), imply $\sum_{n=0}^{\infty} \alpha_{n} b\left(\alpha_{n}\right)<\infty$, and the result follows from Theorem 2. 
Corollary 2. Let $X=L_{p}, p \geqslant 2$ and let $C, T$ and $S$ be as in Theorem 1. Let $\left\{\alpha_{n}\right\}_{n=0}^{\infty}$ be a real sequence satisfying

(i) $0<\alpha_{n}<1, n \geqslant 0$

(ii) $\sum_{n=0}^{\infty} \alpha=\infty$, and

(iii) $\sum_{n=0}^{\infty} \alpha_{n}^{2}<\infty$.

Then, for any $f$ in $C$, the iteration process, $x_{0}$ in $C$,

$$
x_{n+1}=\left(1-\alpha_{n}\right) x_{n}+\alpha_{n} S x_{n}, \quad n \geqslant 0
$$

converges strongly to a solution of the equation $T x=f$ in $C$.

Proof: The proof follows as in the proof of Corollary 1.

ERror Estimates. The sequence $\left\{x_{n}\right\}_{n=0}^{\infty}$ generated in our theorem satisfies

$$
\left\|x_{n}-x^{*}\right\|=O\left(n^{-(s-1) / 2}\right) \text {. }
$$

In particular, if $E=L_{p}, 1<p<\infty$, we have,

and

$$
\left\|x_{n}-x^{*}\right\|=O\left(n^{-(p-1) / 2}\right), \quad \text { if } 1<p \leqslant 2 \text {, }
$$

$$
\left\|x_{n}-x^{*}\right\|=O\left(n^{-1 / 2}\right), \quad \text { if } 2 \leqslant p<\infty
$$

The convergence rates are obtained as in [10], and hence the proof is omitted.

REMARK 3. The only use made of the continuity of $T$ in Theorem 1 and Corollaries 1 and 2 is to obtain the existence of a solution to the equation $T x=f$. Thus, for $C$ and $S$ as in Theorem 2, if $T: C \rightarrow C$ is strongly accretive, and the equation $T x=f$ has a solution $x^{*}$ in $C$, then the iteration process defined in Theorem 1 converges strongly to $x^{*}$.

THEOREM 2. Let $X$ be a real Banach space with uniformly convex dual $X^{*}$, and $C$ a nonempty closed convex and bounded subset of $X$. Let $T: C \rightarrow C$ be a Lipschitz and strongly accretive mapping. For any $f \in C$, define $S: C \rightarrow C$ by,

$$
S x=f+x-T x, \quad \text { for each } x \in C .
$$

Let $\left\{\alpha_{n}\right\}_{n=0}^{\infty}$ and $\left\{\beta_{n}\right\}_{n=0}^{\infty}$ be real sequences satisfying
(i) $0<\alpha_{n}<1, n \geqslant 0$,
(ii) $0 \leqslant \beta_{n} \leqslant \alpha_{n}(1-k)^{2}\left[2 L_{*}\left(1+L_{*}\right)\right]^{-1}, n \geqslant 0$, where $L_{*}=1+L$,
(iii) $\sum_{n=0}^{\infty} \alpha=\infty$ and
(iv) $\sum_{n=0}^{\infty} \alpha_{n} b\left(\alpha_{n}\right)<\infty$ 
Then, for any $f$ in $C$, the iteration process, $x_{0} \in C$,

$$
x_{n+1}=\left(1-\alpha_{n}\right) x_{n}+\alpha_{n} S\left[\left(1-\beta_{n}\right) x_{n}+\beta_{n} S x_{n}\right], \quad n \geqslant 0
$$

converges strongly to a solution of $T x=f$ in $C$.

Proof: The existence of a solution, say $x^{*}$ of $T x=f$, follows from Browder [2] (see also Bogin [1]). Moreover, $x^{*}$ is a fixed point of $S$ and $S$ is Lipschitz with Lipschitz constant $L_{*}=1+L$.

Rewrite (11) as,

$$
x_{n+1}=\left(1-\alpha_{n}\right) x_{n}+\alpha_{n} S y_{n}, \quad n \geqslant 0
$$

where

$$
y_{n}=\left(1-\beta_{n}\right) x_{n}+\beta_{n} S x_{n}, \quad n \geqslant 0 .
$$

Using inequality (6), and equations (12) and (13), we obtain

$$
\begin{aligned}
\left\|x_{n+1}-x^{*}\right\|^{2}= & \left\|\left(1-\alpha_{n}\right)\left(x_{n}-x^{*}\right)+\alpha_{n}\left(S y_{n}-x^{*}\right)\right\|^{2} \\
\leqslant & \left(1-\alpha_{n}\right)^{2}\left\|x_{n}-x^{*}\right\|^{2}+2 \alpha_{n}\left(1-\alpha_{n}\right)\left(S y_{n}-x^{*}, j\left(x_{n}-x^{*}\right)\right\rangle \\
& +\max \left\{\left(1-\alpha_{n}\right)\left\|x_{n}-x^{*}\right\|, 1\right\} \alpha_{n}\left\|S y_{n}-x^{*}\right\| b\left(\alpha_{n}\left\|S y_{n}-x^{*}\right\|\right) \\
\leqslant & \left(1-\alpha_{n}\right)^{2}\left\|x_{n}-x^{*}\right\|^{2}+2 \alpha_{n}\left(1-\alpha_{n}\right)\left(S y_{n}-x^{*}, j\left(x_{n}-x^{*}\right)\right\rangle \\
& +M \alpha_{n} b\left(\alpha_{n}\right),
\end{aligned}
$$

for $M>0$, since $C$ is bounded. That is,

$$
\begin{aligned}
\left\|x_{n+1}-x^{*}\right\|^{2} \leqslant & \left(1-\alpha_{n}\right)^{2}\left\|x_{n}-x^{*}\right\|^{2} \\
& +2 \alpha_{n}\left(1-\alpha_{n}\right)\left(S y_{n}-x^{*}, j\left(x_{n}-x^{*}\right)\right\rangle+M \alpha_{n} b\left(\alpha_{n}\right) .
\end{aligned}
$$

Using inequality (8) we obtain,

$$
\begin{aligned}
\left\langle S y_{n}-x^{*}, j\left(x_{n}-x^{*}\right)\right\rangle & =\left\langle S y_{n}-S x_{n}, j\left(x_{n}-x^{*}\right)\right\rangle+\left\langle S x_{n}-x^{*}, j\left(x_{n}-x^{*}\right)\right\rangle \\
& \leqslant\left\|S y_{n}-S x_{n}\right\|\left\|x_{n}-x^{*}\right\|+(1-k)\left\|x_{n}-x^{*}\right\|^{2} \\
& \leqslant L_{*}\left\|y_{n}-x_{n}\right\|\left\|x_{n}-x^{*}\right\|+(1-k)\left\|x_{n}-x^{*}\right\|^{2} \\
& =L_{*} \beta_{n}\left\|S x_{n}-x_{n}\right\|\left\|x_{n}-x^{*}\right\|+(1-k)\left\|x_{n}-x^{*}\right\|^{2} \\
& \leqslant L_{*} \beta_{n}\left[\left\|S x_{n}-x^{*}\right\|+\left\|x_{n}-x^{*}\right\|\right]\left\|x_{n}-x^{*}\right\| \\
& +(1-k)\left\|x_{n}-x^{*}\right\|^{2} \\
& \leqslant L_{*}\left(1+L_{*}\right) \beta_{n}\left\|x_{n}-x^{*}\right\|^{2}+(1-k)\left\|x_{n}-x^{*}\right\|^{2} .
\end{aligned}
$$

That is,

$$
\left\langle S y_{n}-x^{*}, j\left(x_{n}-x^{*}\right)\right\rangle \leqslant L_{*}\left(1+L_{*}\right) \beta_{n}\left\|x_{n}-x^{*}\right\|^{2}+(1-k)\left\|x_{n}-x^{*}\right\|^{2} .
$$


Using (15) in (14) we obtain

$$
\begin{aligned}
& \left\|x_{n+1}-x^{*}\right\|^{2} \leqslant\left(1-\alpha_{n}\right)^{2}\left\|x_{n}-x^{*}\right\|^{2}+2 \alpha_{n}\left(1-\alpha_{n}\right) L_{*}\left(1+L_{*}\right) \beta_{n}\left\|x_{n}-x^{*}\right\|^{2} \\
& +2 \alpha_{n}\left(1-\alpha_{n}\right)(1-k)\left\|x_{n}-x^{*}\right\|^{2}+M \alpha_{n} b\left(\alpha_{n}\right) \\
& \leqslant\left(1-\alpha_{n}\right)^{2}\left\|x_{n}-x^{*}\right\|^{2}+\alpha_{n}^{2}\left(1-\alpha_{n}\right)(1-k)^{2}\left\|x_{n}-x^{*}\right\|^{2} \\
& +2 \alpha_{n}\left(1-\alpha_{n}\right)(1-k)\left\|x_{n}-x^{*}\right\|^{2}+M \alpha_{n} b\left(\alpha_{n}\right) \text {, using condition (ii) } \\
& \leqslant\left(1-\alpha_{n}\right)^{2}\left\|x_{n}-x^{*}\right\|^{2}+\alpha_{n}^{2}(1-k)^{2}\left\|x_{n}-x^{*}\right\|^{2} \\
& +2 \alpha_{n}\left(1-\alpha_{n}\right)(1-k)\left\|x_{n}-x^{*}\right\|^{2}+M \alpha_{n} b\left(\alpha_{n}\right) \\
& =\left[\left(1-\alpha_{n}\right)^{2}+2 \alpha_{n}\left(1-\alpha_{n}\right)(1-k)+\alpha_{n}^{2}(1-k)^{2}\right]\left\|x_{n}-x^{*}\right\|^{2}+M \alpha_{n} b\left(\alpha_{n}\right) \\
& =\left[1-k \alpha_{n}\right]^{2}\left\|x_{n}-x^{*}\right\|^{2}+M \alpha_{n} b\left(\alpha_{n}\right) \\
& \leqslant\left[1-k \alpha_{n}\right]\left\|x_{n}-x^{*}\right\|^{2}+M \alpha_{n} b\left(\alpha_{n}\right) \text {. }
\end{aligned}
$$

Thus,

$$
\left\|x_{n+1}-x^{*}\right\|^{*} \leqslant\left[1-k \alpha_{n}\right]\left\|x_{n}-x^{*}\right\|^{2}+M \alpha_{n} b\left(\alpha_{n}\right)
$$

and the result follows as in the proof of Theorem 1 .

Corollary 3. Let $X=L_{p}, 1<p \leqslant 2$, and let $C, T$ and $S$ be as in Theorem 2. Let $\left\{\alpha_{n}\right\}_{n=0}^{\infty}$ and $\left\{\beta_{n}\right\}_{n=0}^{\infty}$ be real sequences satisfying

(i) $0<\alpha_{n}<1, n \geqslant 0$

(ii) $0 \leqslant \beta_{n} \leqslant \alpha_{n}(1-k)^{2}\left[2 L_{*}\left(1+L_{*}\right)\right]^{-1}, n \geqslant 0$

(iii) $\sum_{n=0}^{\infty} \alpha_{n}=\infty$, and

(iv) $\sum_{n=0}^{\infty} \alpha_{n}^{p}<\infty$.

Then for any $f$ in $C$, the iteration process $x_{0} \in C$

$$
x_{n+1}=\left(1-\alpha_{n}\right) x_{n}+\alpha_{n} S\left[\left(1-\beta_{n}\right) x_{n}+\beta_{n} S x_{n}\right], \quad n \geqslant 0
$$

converges strongly to a solution of $T x=f$ in $C$.

Proof: The existence of a solution to $T x=f$ follows from Browder [2] (see also Bogin [1]). Remark (2), conditions (iii) and (iv) imply $\sum_{n=0}^{\infty} \alpha_{n} b\left(\alpha_{n}\right)<\infty$. The result follows from Theorem 2.

Corollary 4. Let $E=L_{p}, p \geqslant 2$, and let $C, T$ and $S$ be as in Theorem 1. Let $\left\{\alpha_{n}\right\}_{n=0}^{\infty}$ and $\left\{\beta_{n}\right\}_{n=0}^{\infty}$ be real sequences satisfying

(i) $0<\alpha_{n}<1, n \geqslant 0$

(ii) $0 \leqslant \beta_{n} \leqslant \alpha_{n}(1-k)^{2}\left[2 L_{*}\left(1+L^{*}\right)\right]^{-1}, n \geqslant 0$ 
(iii) $\sum_{n=0}^{\infty} \alpha_{n}=\infty$, and

(iv) $\sum_{n=0}^{\infty} \alpha_{n}^{2}<\infty$.

Then, for any $f$ in $C$, the iteration process, $x_{0} \in C$,

$$
x_{n+1}=\left(1-\alpha_{n}\right) x_{n}+\alpha_{n} S\left[\left(1-\beta_{n}\right) x_{n}+\beta_{n} S x_{n}\right], \quad n \geqslant 0,
$$

converges strongly to a solution of $T x=f$ in $C$.

Proof: The proof follows exactly as in the proof of Corollary 3.

The special case of Theorem 2 for which $\beta_{n}=0$ for all $n \geqslant 0$ yields a special case of Theorem 1 for which $T$ is Lipschitz and strongly accretive.

REMARK 4. The error estimates for Theorem 2 and Corollaries 3 and 4 are as in Theorem 1 and Corollaries 1 and 2.

REMARK 5. It is a consequence of the proofs of Theorems 1 and 2 that, under the hypothesis of the theorems, the solution of the given equation must be unique (see for example [11]).

REMARK 6. If $X$ is a real Banach space with a uniformly convex dual, $X^{*}$, and $C$ a closed, convex, and bounded subset of $X$, our results show that either the Mann or the Ishikawa iteration processes can be used to approximate a solution of the equation $T x=f$ if $T: C \rightarrow C$ is strongly accretive and Lipschitzian. Furthermore, the error estimates obtained for the two methods for this class of nonlinear equations are of the same order, so that for computational purposes, the Mann process may have some advantage due to its simplicity.

Problem. If $X$ is a real Banach space with a uniformly convex dual $X^{*}$, and $C$ a closed, convex and bounded subset of $X$, the result of Theorem 1 shows that the Mann iteration process can be used to approximate a solution of the equation $T x=f$ if $T: C \rightarrow C$ is continuous and strongly accretive. The result of Theorem 2 shows that the Ishikawa iteration process can be used to approximate a solution of the equation if $T: C \rightarrow C$ is Lipschitz and strongly accretive. This leads naturally to the following question.

Can the Ishikawa iteration process be used to approximate a solution of the equation $T x=f$, for any $f$ in $C$, if $T: C \rightarrow C$ is continuous and strongly accretive?

\section{REFERENCES}

[1] J. Bogin, 'On strict pseudo-contractions and a fixed point theorem', in Technion Preprint Series No. MT-219 (Herifer, Israel, 1974). 
[2] F.E. Browder, 'Nonlinear mappings of nonexpansive and accretive type in Banach spaces', Bull. Amer. Math. Soc. 73 (1967), 875-882.

[3] F.E. Browder, 'The solvability of nonlinear functional equations', Duke Math. J. 30 (1963), 557-566.

[4] F.E. Browder, 'Nonlinear equation of evolution and nonlinear accretive operators in Banach spaces', Bull. Amer. Math. Soc. 73 (1967), 470-475.

[5] F.E. Browder, 'Nonlinear monotone and accretive operators in Banach spaces', Proc. Nat. Acad. Sci. U.S.A. 61 (1968), 388-393.

[6] F.E. Browder, 'Nonlinear operators and nonlinear equations of evolution in Banach spaces', Proc. Sympos. Pure Math. 18 (1976).

[7] J. Caristi, 'Fixed point theorems for mappings satisfying inwardness conditions', Trans. Amer. Math. Soc. 215 (1976), 241-251.

[8] C.E. Chidume, 'An approximation method for monotone Lipschitzian operators in Hilbert space', J. Austral. Math. Soc. (Series A) 41 (1986), 59-63.

[9] C.E. Chidume, 'Iterative solution of nonlinear equations of the monotone and dissipative types', Appl. Anal. 33 (1989), 79-86.

[10] C.E. Chidume, 'Fixed point iterations for certain classes of nonlinear mappings, II', J. Nigerian Math. Soc. 8 (1989), 11-23.

[11] C.E. Chidume, 'Iterative solution of nonlinear equations of the monotone type in Banach spaces', Bull. Austral. Math. Soc. 42 (1990), 21-31.

[12] C.E. Chidume, 'An iterative process for nonlinear Lipschitzian strongly accretive mappings in $L_{p}$ spaces', J. Math. Anal. Appl. 151 (1990), 453-461.

[13] K. Deimling, 'Zeros of accretive operators', Manuscripta Math. 13 (1974), 365-374.

[14] W.G. Dotson, 'An iterative process for nonlinear monotonic nonexpansive operators in Hilbert space', Math. Comp. 32 (1978), 223-225.

[15] S. Ishikawa, 'Fixed points by a new iteration method', Proc. Amer. Math. Soc. 149 (1974), 147-150.

[16] T. Kato, 'Nonlinear semigroups and evolution equations', J. Math. Soc. Japan 18/19 (1967), 508-520.

[17] W.A. Kirk, 'A fixed point theorem for mappings which do not increase distance', Amer. Math. Monthly 72 (1965), 1004-1006.

[18] W.R. Mann, 'Mean value methods in iteration', Proc. Amer. Math. Soc. 4 (1953), 506-510.

[19] R.H. Martin, Jr, 'A global existence theorem for autonomous differential equations in Banach spaces', Proc. Amer. Math. Soc. 26 (1970), 307-314.

[20] G.J. Minty, 'Monotone (nonlinear) operators in Hilbert space', Duke Math. J. 29 (1962), 541-546.

[21] C. Morales, 'Surjectivity theorems for multi-valued mappings of accretive type', Comment. Math. Univ. Carolin. 26 (1985).

[22] D. Nevanlinna and S. Reich, 'Strong convergence of contraction semi-groups and of iterative methods for accretive operators in Banach spaces', Israel J. Math. 32 (1976), 44-58. 
[23] W.O. Ray, 'An elementary proof of surjectivity for a class of accretive operators', Proc. Amer. Math. Soc. 75 (1979), 255-258.

[24] S. Reich, 'An iterative procedure for constructing zeros of accretive sets in Banach spaces', Nonlinear Anal. 2 (1978), 85-92.

[25] B.E. Rhoades, 'Comments on two fixed point iteration methods', J. Math. Anal. Appl. 56 (1976).

[26] V.L. Smul'yan, 'On the derivation of the norm in a Banach space', Dokl. Akad. Nauk, SSR 27 (1940), 255-258.

[27] E.H. Zarantonello, 'Solving functional equations by contractive averaging', Technical Report, No. 160 (U.S. Army Math. Centre, Madison Wisconsin 1960).

Department of Mathematics

University of Nigeria

Nsukka

Nigeria 